\title{
Host-Induced Gene Silencing in Barley Powdery Mildew Reveals a Class of Ribonuclease-Like Effectors
}

\author{
Clara Pliego, ${ }^{1}$ Daniela Nowara, ${ }^{2}$ Giulia Bonciani, ${ }^{1}$ Dana M. Gheorghe, ${ }^{1}$ Ruo $\mathrm{Xu},{ }^{3}$ Priyanka Surana, ${ }^{4,5}$ \\ Ehren Whigham, ${ }^{5}$ Dan Nettleton, ${ }^{5}$ Adam J. Bogdanove, ${ }^{5}$ Roger P. Wise, ${ }^{5,6}$ Patrick Schweizer, ${ }^{2}$ \\ Laurence V. Bindschedler, ${ }^{7}$ and Pietro D Spanu ${ }^{1}$ \\ ${ }^{1}$ Department of Life Sciences, Imperial College London, South Kensington Campus, London SW7 2AZ, United Kingdom; \\ ${ }^{2}$ Leibniz-Institute of Plant Genetics and Crop Plant Research, 06466-Gatersleben, Germany; ${ }^{3}$ Department of Statistics, lowa \\ State University, Ames, IA, 50011-1210, U.S.A.; ${ }^{4}$ Bioinformatics and Computational Biology Graduate Program, lowa State \\ University, Ames, IA 50011-3260, U.S.A.; ${ }^{5}$ Department of Plant Pathology \& Microbiology and Center for Plant Responses \\ to Environmental Stresses, lowa State University, Ames, IA, 50011-1020, U.S.A.; ${ }^{6}$ Corn Insects and Crop Genetics Research \\ Unit, U.S. Department of Agriculture-Agricultural Research Service, lowa State University, Ames, lowa 50011-1020, U.S.A.; \\ ${ }^{7}$ Department of Chemistry, University of Reading, Whiteknights Campus, Reading, RG6 6AS, United Kingdom
}

Submitted 7 January 2013. Accepted 20 February 2013.

Obligate biotrophic pathogens of plants must circumvent or counteract defenses to guarantee accommodation inside the host. To do so, they secrete a variety of effectors that regulate host immunity and facilitate the establishment of pathogen feeding structures called haustoria. The barley powdery mildew fungus Blumeria graminis f. sp. hordei produces a large number of proteins predicted to be secreted from haustoria. Fifty of these Blumeria effector candidates (BEC) were screened by host-induced gene silencing (HIGS), and eight were identified that contribute to infection. One shows similarity to $\beta-1,3$ glucosyltransferases, one to metallo-proteases, and two to microbial secreted ribonucleases; the remainder have no similarity to proteins of known function. Transcript abundance of all eight BEC increases dramatically in the early stages of infection and establishment of haustoria, consistent with a role in that process. Complementation analysis using silencing-insensitive synthetic cDNAs demonstrated that the ribonuclease-like BEC 1011 and 1054 are bona fide effectors that function within the plant cell. BEC1011 specifically interferes with pathogen-induced host cell death. Both are part of a gene superfamily unique to the powdery mildew fungi. Structural modeling was consistent, with BEC1054 adopting a ribonuclease-like fold, a scaffold not previously associated with effector function.

Mention of trade names or commercial products in this publication is solely for the purpose of providing specific information and does not imply recommendation or endorsement by the U.S. Department of Agriculture.

Current address for A. J. Bogdanove: Department of Plant Pathology and Plant-Microbe Biology, Cornell University. Ithaca, NY, 14853

Current address for L. V. Bindschedler: School of Biological Sciences, Royal Holloway University of London, Egham, Surrey, TW20 0EX, U.K.

Corresponding author: Pietro D. Spanu; Telephone:+44-207-5945384; Fax: +44-207-5842056; Email: p.spanu@imperial.ac.uk

* The $\boldsymbol{e}$-Xtra logo stands for "electronic extra" and indicates that one supplementary figure and a supplementary data set are published online.

(C) 2013 The American Phytopathological Society
Pathogenic microbes are able to infect plant cells by producing arrays of effectors that manipulate host metabolism and immunity (Dodds and Rathjen 2010). These effectors are usually secreted proteins that act at the host cell surface (Stergiopoulos and de Wit 2009) or are taken up by the plant cell and act internally (Ellis and Dodds 2011). Pathogenic bacteria inject effectors directly into the cell, for example, using the type III secretion system (Hann 2010). The resulting selective pressures for the host to evolve mechanisms that detect effectors and for the pathogen to overcome this detection by effector diversification explain the large arrays of effectors observed in microbial pathogens across the biological spectrum (Haas et al. 2009; Kämper et al. 2006; Takken and Rep 2010).

Plants have exquisitely sensitive systems for the detection of microbial invaders (Boller and Felix 2009). The action of successful pathogen effectors is clear in biotrophic interactions, in which the microbe colonizes tissues and cells while avoiding or suppressing rejection and host death. This is particularly evident in the obligate biotrophs, such as rusts and powdery mildews. For example, the cereal powdery mildew, Blumeria graminis, penetrates living epidermal cells and, in compatible interactions in which disease is eventually manifested, a peg derived from a surface appressorium penetrates the cell wall and develops a multidigitate haustorium surrounded by a hostderived membrane (Glawe 2008). The host cell, which may be penetrated by many haustoria simultaneously, remains alive and provides nutrients to the fungus in order for it to produce the masses of conidia, visible as the eponymous "powder", on the plant surface (Both and Spanu 2004).

Effector discovery in the barley powdery mildew fungus $B$. graminis f. sp. hordei has been driven by the identification of i) genes specifically upregulated during infection (Both et al. 2005), ii) RNAs specifically associated with haustoria (Godfrey et al. 2010; Spanu et al. 2010), iii) proteins found specifically in haustoria (Bindschedler et al. 2009, 2011), iv) genes predicted to encode small secreted proteins with no evident homologs in other fungi by means of a bioinformatic survey of the B. graminis f. sp. hordei genome (Pedersen et al. 2012), and v) characterization of avirulence genes in the fungus that confer race-specific incompatibility on barley genotypes with corresponding resistance genes (Ridout et al. 2006; Sacristán et al. 2009).

Vol. 26, No. 6, 2013 / 633 


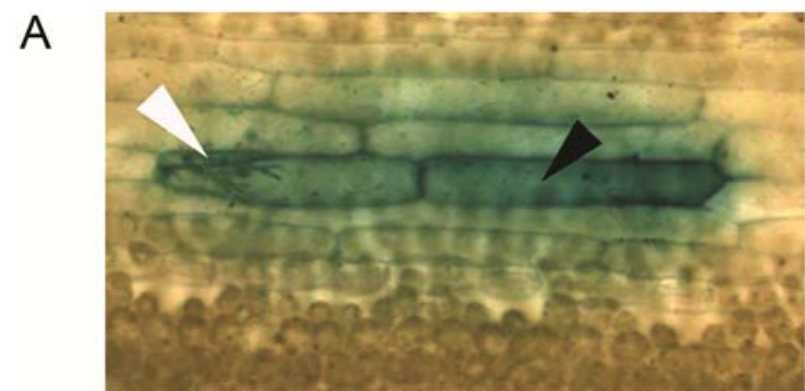

B

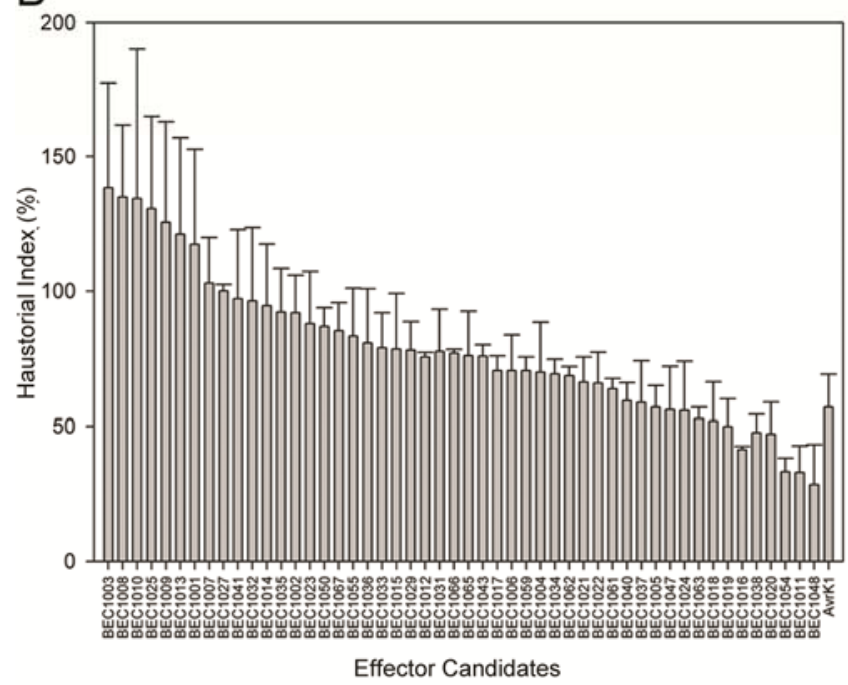

Fig. 1. Functional analysis of 50 Blumeria graminis f. sp. hordei effector candidate (BEC) genes carried out by host-induced gene silencing. A, Infected barley leaves stained to reveal $\beta$-glucuronidase (GUS) activity and haustorial development. Examples are shown of GUS-positive epidermal cells with fully developed haustoria (white arrow) or of GUS-positive cells with no haustoria (black arrow). The cells with partial staining surrounding the GUS-positive cells were not counted. B, Barley leaves were cobombarded with the GUS reporter gene and an RNAi construct directed against each candidate effector gene, followed by inoculation with $B$. graminis $\mathrm{f}$. sp. hordei 3 days later. The $B$. graminis infection rate is given as the haustorium index (HI), calculated as the number of haustoria in GUS-expressing cells divided by the total number of GUS-expressing cells. HI calculations were referred to the empty RNAi-vector control (pIPKTA30) set up to $100 \%$. Bars represent the average of three independent experiments carried out for each BEC.
The lack of a robust and reproducible stable transformation method for any powdery mildew fungus hampers genetic validation of candidate effectors. This difficulty has been alleviated by the development of host-induced gene silencing (HIGS) (Nowara et al. 2010). In barley, this is achieved by particle bombardment of epidermal cells to introduce and transiently express constructs encoding hairpin RNAs corresponding to targeted B. graminis transcripts; the epidermis is then inoculated, and the effect on pathogen development is observed by measuring the haustorial index (HI), i.e., the frequency of full haustorial development relative to empty-vector controls. HIGS of the $B$. graminis f. sp. hordei avirulence genes $A V R_{K 1}$ and $A V R_{10}$ reduced the $\mathrm{HI}$ in plants lacking the cognate resistance genes, demonstrating a role in infection for these effectors. In the same study, silencing of two fungal $\beta 1,3$-glucanosyltransferases that are coordinately upregulated during the early infection stages (Both et al. 2005) similarly decreased powdery mildew development (Nowara et al. 2010). Used increasingly to investigate a number of plant-pathogen interactions, it is assumed that HIGS is mediated by specific RNA interference (RNAi) driven by double-stranded RNA transferred from the host to the closely interacting pathogen, but the precise mechanism is not yet understood (Nunes and Dean 2012).

B. graminis f. sp. hordei produces an array of proteins specifically associated with infected host tissues (Pedersen et al. 2012). Fifty of these B. graminis f. sp. hordei effector candidates (BEC) were screened by HIGS, and eight were identified that, when silenced, resulted in significantly decreased pathogen development. The eight BEC represent a diversity of predicted molecular structures and functions, including two with similarity to fungal RNAses. One of the RNAse-like proteins interfered specifically with pathogen-induced host cell death.

\section{RESULTS}

\section{HIGS-based identification}

\section{of BEC that contribute to infection.}

We initially selected a panel of 67 BEC, designated BEC1001 to BEC1067 (Supplementary Dataset S1). The majority of these encode proteins identified by mass spectrometry as associated specifically with haustoria (Bindschedler et al. 2009, 2011). Others encode small secreted proteins that were found to be highly upregulated in infected epidermis containing haustoria (Spanu et al. 2010). Of the 67 BEC genes, 63 encode proteins

Table 1. Significant Blumeria graminis f. sp. hordei effector candidates resulting from the HIGS (host-induced gene silencing) screen

\begin{tabular}{|c|c|c|c|c|c|c|c|}
\hline \multirow[b]{2}{*}{ BEC $^{\mathbf{a}}$} & \multicolumn{2}{|c|}{ GUS cells $^{b}$} & \multirow[b]{2}{*}{ Haustorial index ${ }^{c}$} & \multirow[b]{2}{*}{ Odds ratio $^{d}$} & \multirow[b]{2}{*}{ Adjusted $P$ value } & \multicolumn{2}{|c|}{$95 \%$ Confidence interval of odds ratio } \\
\hline & Total & With haustoria & & & & Lower limit & Upper limit \\
\hline pTA30 & 8,052 & 802 & $100.00 \%$ & & & & \\
\hline 1011 & 1,453 & 43 & $29.71 \%$ & 0.3086 & $<0.0001$ & 0.1433 & 0.6646 \\
\hline 1054 & 1,785 & 73 & $41.06 \%$ & 0.4172 & $<0.0001$ & 0.2286 & 0.7612 \\
\hline$A V R_{K I}$ & 3,323 & 187 & $56.50 \%$ & 0.5697 & 0.0002 & 0.3818 & 0.8500 \\
\hline $1038^{K I}$ & 2,165 & 121 & $56.11 \%$ & 0.5785 & 0.0145 & 0.3523 & 0.9498 \\
\hline 1016 & 1,366 & 69 & $50.71 \%$ & 0.5059 & 0.0151 & 0.2724 & 0.9397 \\
\hline 1005 & 1,294 & 63 & $48.88 \%$ & 0.4921 & 0.0174 & 0.2566 & 0.9436 \\
\hline 1019 & 1,698 & 88 & $53.03 \%$ & 0.5437 & 0.0184 & 0.3100 & 0.9536 \\
\hline 1040 & 1,979 & 115 & $58.34 \%$ & 0.6054 & 0.0478 & 0.3672 & 0.9980 \\
\hline 1018 & 1,520 & 87 & $57.47 \%$ & 0.5736 & 0.0568 & 0.3268 & 1.0064 \\
\hline
\end{tabular}

${ }^{\mathrm{a}} \mathrm{BEC}=$ Blumeria graminis f. sp. hordei effector candidates

${ }^{\mathrm{b}}$ GUS ( $\beta$-glucuronidase) cells with haustoria. Raw numbers indicate the combined total of five to 12 (or 34 for the pTA30 control) replicates, seven leaves per replicate.

${ }^{c}$ Haustorial index (HI) is the ratio of GUS cells with haustoria divided by total GUS cells. The control (pTA30) is set to $100 \%$ and the others are scaled according to the following formula: $\mathrm{HI}_{\text {Scaled }}=\left(1 / \mathrm{H}_{\text {IRaw, pTA30 }}\right) \times \mathrm{HI}_{\text {Raw, BEC }}$.

${ }^{\mathrm{d}}$ Odds ratio $=\left(\mathrm{p}_{1} /\left(1-\mathrm{p}_{1}\right)\right) /\left(\mathrm{p}_{2} /\left(1-\mathrm{p}_{2}\right)\right)$, where $\mathrm{p}_{1}$ and $\mathrm{p}_{2}$ are the probability of haustoria formation in BEC and control, respectively. An odds ratio of more than one suggests that silencing of the BEC increased susceptibility, while an odds ratio of less than one suggests that silencing of the BEC increased resistance.

${ }^{\mathrm{e}}$ Adjusted $P$ values (pTA30 vs. others) were obtained using a generalized linear mixed effects model to test for a significant difference between the BEC and the pTA30 control. BEC with an adjusted $P$ value $\leq 0.05$ had odds of haustoria formation significantly different from control. 
with a predicted signal peptide but no transmembrane domain in the mature protein (SignalP V4.0 [Petersen et al. 2011; Quevillon et al. 2005]). Likely to be secreted, these proteins were considered as especially strong candidate effectors.

Fifty of the selected 67 BEC cDNAs were then isolated by designing primers corresponding to the predicted mature (processed) coding sequences and amplifying them by reverse-transcription polymerase chain reaction (RT-PCR). The template cDNA was synthesized on RNA extracted from infected barley leaves 5 days after inoculation. The products of amplification were cloned into the Gateway entry vector pCR8 and were sequenced for quality control.

The BEC cDNAs were then transferred into the destination vector pTA30, designed for transient gene-silencing experiments (Douchkov et al. 2005) such as HIGS. pTA30 drives the expression of an inverted repeat RNA predicted to form a hairpin (double-stranded) molecule. In the first round of screening, the resulting silencing constructs for each of the $50 \mathrm{BEC}$ were co-bombarded into barley leaves together with the pUbiGUS reporter plasmid, which acted as a transfection reference marker. The leaves were left for 3 days to give the epidermal cells time to recover and express the RNAi constructs, and they were then inoculated with $B$. graminis. Two days after inoculation, $\beta$-glucuronidase (GUS) activity was assayed histochemically and haustorial development scored by microscopy. GUS-positive cells with a well-defined haustorial body were scored as having developed a haustorium (Fig. 1A). An $A V R_{K 1^{-}}$ RNAi construct was used as a positive control for effectors that might reduce HI (Nowara et al. 2010). pTA30 without an insert was used as a negative control. HI observed following
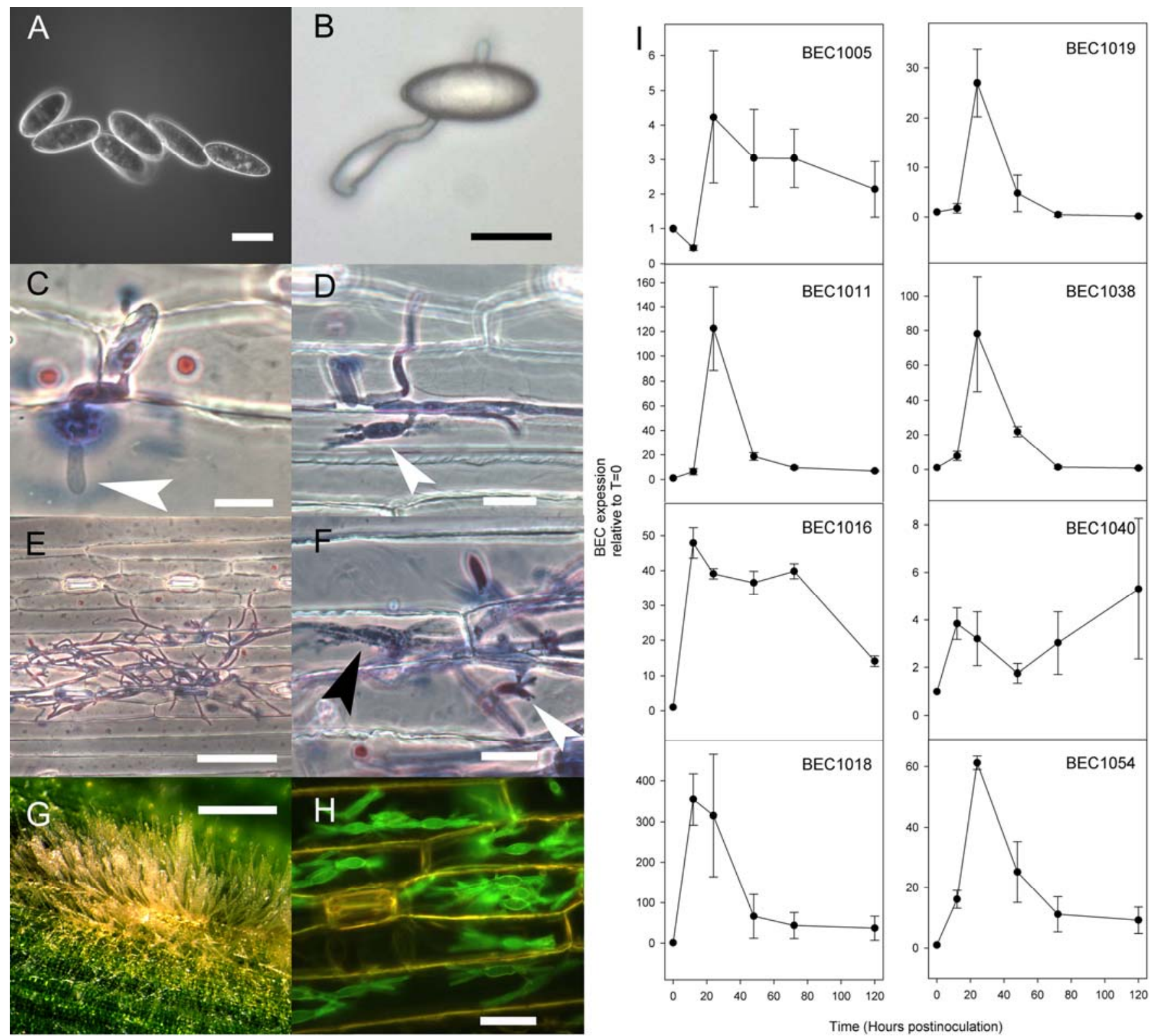

Fig. 2. Expression of Blumeria graminis f. sp. hordei effector candidate (BEC) transcripts during early stages of infection. A to $\mathbf{H}$, The development of Blumeria graminis at the times assayed here. A, Ungerminated conidia ( $0 \mathrm{~h}$ postinoculation [hpi], bar $20 \mu \mathrm{m}) ; \mathbf{B}, 12 \mathrm{hpi}$; C, 24 hpi, showing haustorial primordia (arrow, bar $20 \mu \mathrm{m}) ; \mathbf{D}, 48 \mathrm{hpi}$, with young but functional haustoria (arrow, bar $40 \mu \mathrm{m}$ ); $\mathbf{E}$ and $\mathbf{F}, 72 \mathrm{hpi}$ but functional haustoria (arrows; bars = $100 \mu \mathrm{m}$ [E]) and $40 \mu \mathrm{m}[\mathrm{F}]) ; \mathbf{G}, 5 \mathrm{dpi}$, fully formed colony $(\mathrm{bar}=0.2 \mathrm{~mm}) ; \mathbf{H}, 5 \mathrm{dpi}$, mature haustoria showing multiple haustoria in individual epidermal cells $($ bar $=$ $20 \mu \mathrm{m})$. I, Time course infection stages surveyed in the analysis are ungerminated conidia ( 0 hpi) and 12, 24, 48, 72, and 120 hpi on barley leaves. Reversetranscription quantitative polymerase chain reaction expression levels were normalized with $\beta$ tubulin gene (GenBank number HM538443.1). The normalized values are expressed relative to those of RNA in ungerminated conidia. Error bars are standard deviation of three independent biological replicates. 
silencing of the 50 BEC ranged from approximately 140 (BEC1003) to 30\% (BEC1011), indicating a positive role of the BEC in penetration and haustorial formation (Fig. 1B).

We selected the $18 \mathrm{BEC}$ (in addition to $A V R_{K 1}$ ) for which silencing constructs caused the greatest reduction of $\mathrm{HI}$ for the first three independent trials. None of the apparent increases in HI caused by some of the other BEC was statistically significant, based on initial screening, and these were not examined further. In order to increase statistical power, we then performed up to four additional trials for each of the $18 \mathrm{BEC}$. These results were combined with those of the first screen, and a generalized linear mixed-effects model was fit to the haustoria counts. For each leaf, the number of GUS cells with haustoria was modeled as a binomial random variable with the total number of GUS cells as the number of trials, and a success probability (probability of forming haustoria) specific to each combination of experiment and BEC (discussed below). As shown in Table 1, the odds of haustoria formation for a BEC divided by the odds of haustoria formation for the control was estimated for each BEC. In addition to $A V R_{K 1}$, eight BEC constructs were found to impart a statistically significant decrease on HI. Importantly, BLAST searches revealed no similarity of any of the eight BEC to any barley sequence in GenBank or to any other sequences in the barley (International Barley Genome Sequencing Consortium 2012). This observation strongly suggests that the HIGS effect observed for each BEC was due to silencing of the fungal gene rather than a barley transcript, and that the eight BEC indeed contribute to infection.

\section{Expression of the BEC during infection.}

We measured the expression levels of all eight BEC genes by RT-quantitative (q)PCR. RNA was extracted from ungerminated conidia $(0 \mathrm{~h}$ postinoculation [hpi]); Fig. $2 \mathrm{~A})$ and from infected barley leaves $12,24,48,72$, and 120 hpi. This time course covers all stages of initial infection, i.e., formation of a functional appressorium (12 hpi; Fig. 2B), penetration and formation of the first haustorium primordia (24 hpi; Fig. 2C), establishment of fully functional haustoria and extension of epiphytic colonies on the surface of the epidermis (48 hpi; Fig. 2D), expansion of colonies (72 hpi; Fig. 2E) and the formation of many secondary haustoria (Fig. 2F), and finally, the development of erect sporulating conidiophores and the establishment of multiple haustoria in many host cells (Fig. 2H). At 12, 24 , and $48 \mathrm{hpi}$, when the amount of fungus is low relative to the plant, RNA was isolated from epidermal peels rather than whole-leaf segments, in order to enrich for fungal RNA. The levels of expression were normalized relative to the $B$. graminis $\beta$-tubulin (tub2) gene (GenBank number HM538443.1). The specific RNA content was then calculated relative to the abundance of candidate effector RNA in ungerminated conidia set arbitrarily at 1.0 (Fig. 2I). The accumulation of BEC1005 transcripts initially decreased to 0.45 by $12 \mathrm{hpi}$, as compared with the $t u b 2$ control, increased three- to fourfold and remained at that level for 3 days, and then, decreased to twofold by day 5 . BEC1011 transcripts increased sixfold at 12 hpi and 120-fold in the first $24 \mathrm{~h}$, but returned to about 20- to 10-fold conidial levels thereafter. BEC1016 and BEC1018 transcripts increased quickly, reached a maximal level of 50-fold and 350-fold at 12 hpi, respectively, remained relatively high for 3 days, and then decreased after 5 days. BEC1019, BEC1038, and BEC1054 all displayed very similar expression profiles, increased at $24 \mathrm{hpi}$ (about 27-, 80- and 60-fold respectively), and then decreasing gradually to conidial levels (BEC1019 and 1038) or to about 10-fold (BEC1054) 5 days after inoculation. BEC1040 RNA increased moderately by $12 \mathrm{hpi}$, decreased to the levels seen in conidia by 48 hpi and then increased again, however the fold induction was moderate compared to other BEC.
BEC1005, BEC1019, and BEC1054 are similar

to glucanase, metalloprotease, and

ribonuclease-like proteins.

BEC1005 encodes a 299-amino acid preprotein that is predicted to be processed and secreted as a 281 -amino acid mature protein. It is homologous with $\beta 1,3$-endoglycosidases of the CAZy database (Cantarel et al. 2009) glyco_hydrolase 17 superfamily. The most significant BLAST hits were to proteins predicted in Sclerotinia sclerotiorum and Botrytis cinerea (both e $<10^{-80}$ ) and other genes encoding similar proteins in many filamentous ascomycete fungi and yeasts but differs from the two $\beta 1,3$-endoglycosidases previously found to be important in B. graminis pathogenicity (Nowara et al. 2010).

BEC1019 is conserved in a large number of ascomycetes. It encodes a 316-amino acid preprotein that is predicted to be processed and secreted as a 298-amino acid mature protein. Like BEC1005, the most significant BLAST hits were to homologs in Sclerotinia sclerotiorum $\left(\mathrm{e}<10^{-96}\right)$ and Botrytis cinerea $\left(\mathrm{e}<10^{-93}\right)$ and identified this candidate effector as a member of the clan peptidase MA, which include the closely related M35 peptidase family.

The other six BEC showed no informative sequence similarities. We therefore attempted structural predictions for clues to function using the Phyre ${ }^{2}$ server using the 'Normal Modelling' option (Kelley and Sternberg 2009). This software predicts three-dimensional protein structures using homology modeling. Thus, a given sequence can have certain regions modeled with high confidence and other regions modeled with low confidence depending on homology of those regions to known proteins. Indeed, a 259-amino acid region of BEC1005 (Ser20 to Trp279) was modeled with $100 \%$ confidence onto an endo 31-3 glucanase structure. Similarly, 184 amino acids from Gly54 to Ala238 of BEC1019 were modeled with a $100 \%$ confidence onto a metalloprotease structure (a fungal zinc peptidase family). For the BEC with no informative sequence similarities, structural predictions using the Phyre $^{2}$ server revealed that Cys27 to Gly108 of BEC1054 could be modeled with $42.9 \%$ confidence onto a fungal ribonuclease structure. None of the other candidate effectors returned structural models with confidence higher than $40 \%$ for any length of the protein and were, therefore, considered nonsignificant. BEC1011, however, shares $75 \%$ nucleotide identity with BEC1054 (Supplementary Fig. S1A). Although the divergence of BEC 1011 protein sequence prevented high confidence modeling on the fungal RNAse, it is clearly paralogous with BEC1054 and is likely to adopt the same fold.

\section{BEC1011 and BEC1054 are bona fide effectors that function inside the plant cell.}

HIGS constructs for BEC1011 and BEC1054 caused the greatest effect on disease development, each resulting in a 60 to $70 \%$ decrease (Table 1 ; adjusted $P$ value $<0.0001$ ). We therefore chose to characterize these BEC further. Although no barley sequences with similarity to these BEC were detected, as noted above, we first sought to experimentally confirm that the effect of the RNAi constructs for each was, in fact, due to silencing of the fungal gene. Because technical limitations preclude distinguishing BEC transcript levels in haustoria inhabiting host cells expressing the silencing construct, from those haustoria inhabiting cells not expressing the silencing construct within the same leaf sample, we took a complementation approach to validation of BEC1011 and BEC1054 function. We generated synthetic BEC1011 and BEC1054 clones with silent ("wobble") mutations that minimize sequence identity to the wild-type cDNAs while retaining favorable codon usage for expression in barley so they would be insensitive to the corresponding silencing constructs. Importantly, we excluded the $5^{\prime}$ sequences 
encoding the predicted signal peptide of each BEC. The resulting BEC1011- and BEC1054-wobble sequences are 61 and $67 \%$ identical to the original BEC1011 and BEC1054 sequences, respectively. These wobble sequences were subcloned into the expression vector pIPKTA9 (Zimmermann et al. 2006). For each BEC, a HIGS complementation assay was then performed, in which the pIPKTA9-BEC wobble constructs were co-bombarded into barley with the corresponding RNAi silencing vector for that BEC. This was carried out in five independent replicates. As described above for the general BEC screen, haustoria count data were fit using a generalized linear mixed effects model.

As illustrated in Table 2, expression of the RNAi:1011 or RNAi:1054 silencing constructs alone reduced the odds of haustoria formation to 0.31 and 0.42 times the pTA30 control, respectively. Neither of the wobble:1011 and wobble:1054 expression constructs alone significantly altered the odds of haustoria formation ( 0.82 and 0.85 times the control, respectively). When the RNAi silencing plasmids were co-bombarded with their respective wobble constructs, the odds of haustoria formation were not significantly different from the controls $(0.92$ and 0.99 for BEC1011 and BEC1054, respectively), indicating functional complementation of each silenced BEC gene by its corresponding wobble construct. Thus, the decrease in HI caused by each silencing construct is, indeed, due to silencing of the BEC. These results establish BEC1011 and BEC1054 as bona fide effectors. Furthermore, barring the unlikely transfer of intact wobble transcript into the fungal cell and its expression there, the results indicate that BEC1011 and BEC1054 function inside the plant cell.

\section{BEC1011 interferes with host cell death.}

An essential function of effectors is to suppress host programmed cell death (PCD) that occurs during the defense response upon pathogen recognition (Wang and Huang 2011). To determine if the contributions of any of the eight candidate effectors to infection related to an ability to suppress host PCD, we took advantage of the observation that transient expression of maize transcription factor genes $B$-Peru and $C 1$ in barley leads to accumulation of anthocyanins and that this only occurs in intact vacuoles of viable cells (Schweizer et al. 2000). To assess effects of silencing the BEC on the fractions of host cells that die during the early stages of infection, we co-bombarded each of the RNAi BEC constructs with the $B$-Peru/C1expression plasmid pBC17 and pUbiGUS (as cell-death insensitive marker for transformation efficiency). Co-bombardment of pBC17 with empty vector pTA30 and pUbiGUS served as a control. After counting the number of cells that had accumulated red anthocyanin pigments (Fig. 3A), the leaves were stained for GUS activity and the number of GUS positive cells was determined for normalization (Fig 3B). The empty vector control (pTA30) resulted in a ratio of normalized anthocyaninstained cells in the infected versus the noninfected leaves of 1.3 (hereafter the referred to as the anthocyanin ratio). The anthocyanin ratios obtained from the co-bombardments with all effector RNAi constructs did not differ from the control except for the RNAi construct targeting BEC1011. In the case of RNAi of BEC1011, the anthocyanin ratio dropped to an average 0.46 (standard deviation: 0.09). This indicated that under these conditions, BEC1011 suppresses host cell death. To what extent the strongly reduced $\mathrm{HI}$ in cells expressing the BEC1011 RNAi construct was due to increased cell death upon $B$. graminis attack remains unclear because we do not know the kinetics of cell-death triggering. If it happened after the establishment of the first haustorium approximately 24 hai, it would not contribute to HI reduction. In that case, HI might underestimate final effect of the corresponding RNAi construct on the final outcome of the interaction including sporulation.

\section{DISCUSSION}

Plant pathogenic fungi and oomycetes appear to possess vast arrays of genes encoding proteins that bear the hallmark of effectors (Stergiopoulos and de Wit 2009). The biotrophic pathogens, which must ensure that host cells and tissues remain alive during the critical infection stages, are no exception. For example, the nonobligate biotroph Ustilago maydis has 494 putative effectors, often arranged in clusters. These are largely conserved in the closely related Sporisorium reilianum (Schirawski et al. 2010). In the obligate rust Melampsora larici-populina, 1,184 genes encoding small secreted proteins were identified and characterized as likely to be effectors (Hacquard et al. 2012). Likewise, in B. graminis, we have found a superfamily of 491 genes encoding small proteins, predicted to be secreted and with features typical of effectors (Pedersen et al. 2012). These candidate secreted effector proteins (CSEP) differ from previously identified $B$. graminis avirulence proteins $\mathrm{AVR}_{\mathrm{A} 10}$ and $\mathrm{AVR}_{\mathrm{K} 1}$, which do not contain a predicted signal peptide (Ridout et al. 2006; Sacristán et al. 2009). The CSEP superfamily includes many of the genes and proteins previously identified as specifically expressed in haustoria (Godfrey et al. 2010) and in infected epidermis (Bindschedler et al. 2009, 2011). One member of this superfamily, CSEP0055, has recently been shown to reduce HI when silenced by HIGS and interacts with barley pathogenesis-related protein PR17c and is, therefore, an experimentally validated effector (Zhang et al. 2012). Apart from this protein, however, the majority of candidate effectors of $B$. graminis have yet to be characterized.

Table 2. Results of combinations of silent ("wobble") mutations and RNA interference (RNAi) on formation of haustoria by Blumeria graminis f. sp. hordei

\begin{tabular}{|c|c|c|c|c|c|c|c|}
\hline \multirow[b]{2}{*}{ Wobble constructs } & \multicolumn{2}{|c|}{ GUS cells ${ }^{\mathrm{a}}$} & \multirow[b]{2}{*}{ Haustorial index ${ }^{b}$} & \multirow[b]{2}{*}{ Odds ratio $^{c}$} & \multirow[b]{2}{*}{$P$ value $^{\mathrm{d}}$} & \multicolumn{2}{|c|}{ 95\% Confidence interval of odds ratic } \\
\hline & Total & With haustoria & & & & Lower limit & Upper limit \\
\hline pTA30/pIPKTA9/pUbiGUS & 4,191 & 405 & $100.00 \%$ & & & & \\
\hline RNAi:1011 & 2,330 & 113 & $50.19 \%$ & 0.4999 & $<0.0001$ & 0.3409 & 0.7330 \\
\hline RNAi: 1054 & 1,855 & 67 & $37.38 \%$ & 0.3735 & $<0.0001$ & 0.2332 & 0.5981 \\
\hline Wobble:1011/RNAi:1011 & 1,409 & 126 & $92.54 \%$ & 0.9222 & 0.9988 & 0.6344 & 1.3407 \\
\hline Wobble:1011 & 1,603 & 128 & $82.63 \%$ & 0.8183 & 0.6867 & 0.5658 & 1.1838 \\
\hline Wobble: 1054 & 1,585 & 131 & $85.53 \%$ & 0.8547 & 0.8891 & 0.5924 & 1.23 \\
\hline Wobble:1054/RNAi:1054 & 1,863 & 178 & $98.87 \%$ & 0.9886 & 1.0000 & 0.7111 & 1.3744 \\
\hline
\end{tabular}

${ }^{a}$ GUS ( $\beta$-glucuronidase). Raw numbers indicate the combined total of five (or 13 for the controls) replicates, seven leaves per replicate.

${ }^{\mathrm{b}}$ Haustorial index (HI) is the ratio of GUS cells with haustoria divided by total GUS cells. The control (pTA30) is set to 100\% and the others are scaled according to the following: $\mathrm{HI}_{\text {Scaled }}=\left(1 / \mathrm{HI}_{\text {Raw, } \mathrm{pTA} 30}\right) \times \mathrm{HI}_{\mathrm{Raw}, \mathrm{BEC}}$.

${ }^{\mathrm{c}}$ Odds ratio $=\left(\mathrm{p}_{1} /\left(1-\mathrm{p}_{1}\right)\right) /\left(\mathrm{p}_{2} /\left(1-\mathrm{p}_{2}\right)\right)$, where $\mathrm{p}_{1}$ and $\mathrm{p}_{2}$ are the probability of haustoria formation in BEC and control, respectively.

d Adjusted $P$ values (pTA30 vs. others) were obtained using a generalized linear mixed effects model to test for a significant difference between the BEC and the pTA30 control. BEC with an adjusted $P$ value $\leq 0.05$ had odds of haustoria formation significantly different from control.

e Controls were co-bombarded with empty pTA30, empty pIPKTA9, and pUbiGUS vectors. 
In this paper, we report the HIGS-based functional analysis of 50 genes encoding BEC. These genes encode proteins we had previously identified as abundant in infected tissues (Bindschedler et al. 2009, 2011) and which showed the greatest levels of differential transcript accumulation in infection structures (Spanu et al. 2010).

The use of HIGS to identify B. graminis genes that contribute towards pathogenic development was introduced and validated by Nowara and colleagues (2010) and has also been used as a functional assay in other fungal pathosystems (Nunes and Dean 2012). We chose to measure the impact of HIGS of BEC on HI. We anticipated identifying effectors that are critical to the initial development of the first haustoria. Nevertheless, across BEC, we observed HIGS effects ranging from a nonsignificant increase in $\mathrm{HI}$ to a significant reduction in HI. In this report, we focused on the eight candidate effectors whose silencing led to a significant and reproducible reduction in HI. It is likely that others exist that are important later in the interaction, in more subtle ways, or redundantly, yet escaped our screen.

The expression profiles of the eight BEC analyzed here showed that all candidate effectors were upregulated relative to the $B$. graminis $\beta$-tubulin during infection. We identified four distinct trends. The most common, observed with BEC1011, BEC1019, BEC1038, and BEC1054, showed a clear maximum
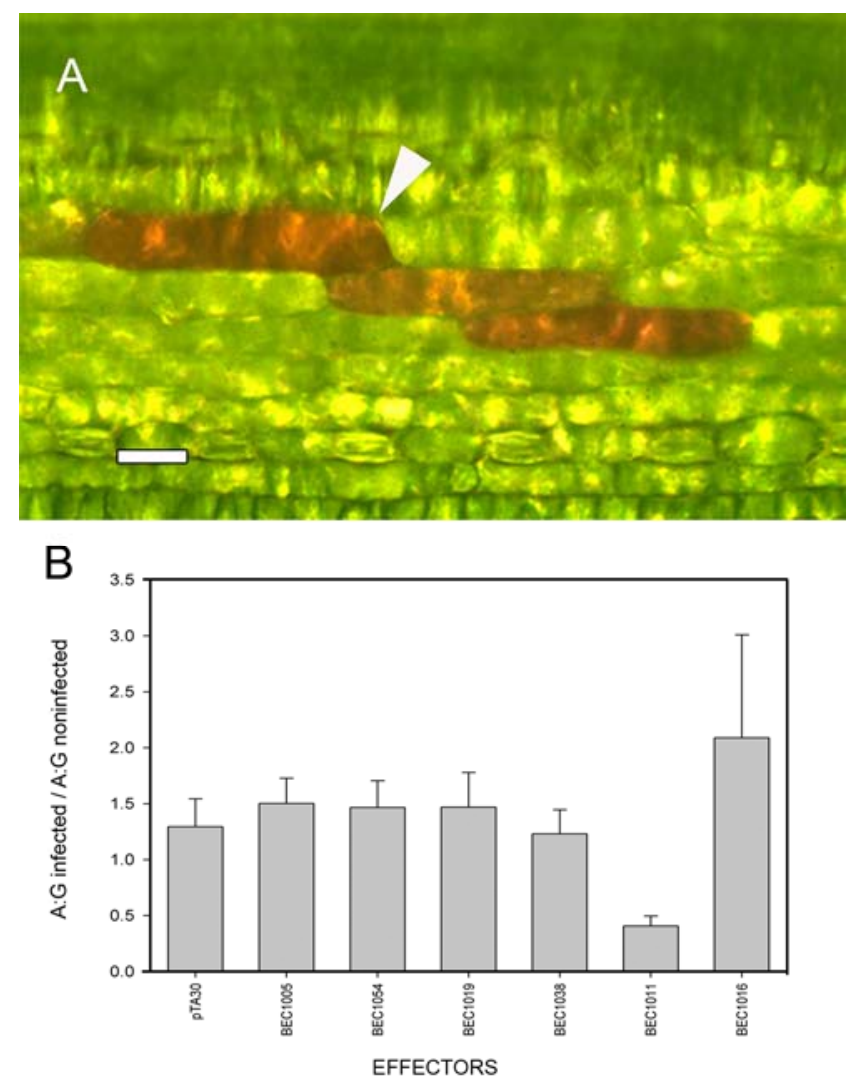

Fig. 3. BEC1011 interferes with pathogen-induced cell death. Barley leaves were co-bombarded with pUbiGus as a transformation marker, pTA30BEC, for silencing selected Blumeria graminis f. sp. hordei effector candidates (BEC), and the B-Peru/C1-expression plasmid pBC17 that induces anthocyanin accumulation in barley epidermal cells. A, Unstained barley leaf showing epidermal cells that accumulate anthocyanins (arrow; bar $=20 \mu \mathrm{m}$ ) 5 days after bombardment. B, Quantification of the relative numbers of anthocyanin-producing cells measured as the ratio of anthocyanin-accumulating cells relative to the number of $\beta$-glucuronidase (GUS) expressing cells $(\mathrm{A}: \mathrm{G})$ in the presence and absence of Blumeria graminis $\mathrm{f}$. $\mathrm{sp}$. hordei. Co-transformation of $\mathrm{pBC} 17$ with empty vector pIPKTA30 served as control. Values are means of three independent experiments $(B a r=$ standard errors of the means). at $24 \mathrm{hpi}$, which immediately precedes formation of the first mature haustoria. This suggests that the role played by these candidate effectors may be most important in the earliest stages of penetration. The second trend, observed with BEC1016 and BEC1018, was characterized by a dramatic early increase and a sustained upregulation throughout the time course. This early response may be indicative of a role before actual cell penetration, perhaps even as early as in primary germ-tube formation (Hoefle and Huckelhoven 2008). The sustained high levels of expression hint at an important function also in later stages of colony development, perhaps during formation of secondary haustoria. The HIGS assay used here cannot discriminate this possibility. A third pattern, that of BEC1005 expression, showed an increase only after $12 \mathrm{hpi}$, suggesting a role in later development of one or both the infection or haustoria. The final trend observed was for BEC1040, which showed a moderate increase relative to ungerminated spores at all stages of fungal development. Across all four patterns, the common increase in candidate effector RNA accumulation during the overall time course is consistent with the observed reduction in $\mathrm{HI}$ resulting from HIGS; silencing of the candidate effectors between 0 to 24 hpi is likely to have the greatest impact if increased candidate effector RNA transcript is necessary at this stage.

Sequence and predicted structure-based similarities revealed possible functions for four of the eight BEC that suggest a diversity of roles in infection. BEC1005 has significant sequence similarity to fungal glucosyl transferases. In yeast, the homologous BGL2 protein is involved in cell-wall glycan remodeling (Goldman et al. 1995). Intriguingly, in Aspergillus fumigatus, deletion of glucosyl transferases GEL2 results in altered morphology and reduced infection of mice (Mouyna et al. 2005). BEC1005, therefore, may play an important role in the substantial cell-wall remodeling that occurs during formation of the branched haustorium. Thus, it may best be considered a candidate morphogenetic factor (albeit central for pathogenesis) rather than an effector in the strict sense.

BEC1019 was predicted by structural modeling to be related to the M35 peptidase family. BEC1019 has a high sequence similarity to the Aspergillus fumigatus major allergen Asp F2, which binds immunoglobulin $\mathrm{E}$ in humans with allergic bronchopulmonary aspergillosis (Banerjee et al. 1997). AspF2 sequence is also closely related to members of the M35 peptidase family. Based on these apparent similarities, BEC1019 might be involved in protein degradation, nutrition, direct penetration of the cell walls, or interference with host receptors that sense the pathogen.

Structural prediction also revealed that BEC1054 is similar to microbial RNAses. This similarity has been found in many of the B. graminis CSEP described by Pedersen and colleagues (2012). Though the protein sequence of BEC1011 was too divergent to be modeled directly on a microbial RNAse, it showed clear sequence-based paralogy with BEC1054, indicating that it, too, is a member of this large protein family.

The absence of sequence similarity of BEC1054 and BEC1011 with any barley genomic sequence suggests that the likelihood of an off-target effect against a host gene is low. Nevertheless, we controlled for this possibility by assessing the ability of alternate BEC1054 and BEC1011 coding constructs with no possible targeting by the RNAi constructs (Dong et al. 2006; Nowara et al. 2010) to complement the phenotypes that resulted from the HIGS constructs. Co-bombardment of these wobble constructs with their respective HIGS constructs did not affect the HI, indicating that the effect of the HIGS constructs alone on $\mathrm{HI}$ is, indeed, dependent on the direct silencing of the respective BEC. The fact that the expression constructs restore HI to normal levels in the presence of 
the respective HIGS constructs, taken together with the apparently distinct functions of BEC1054 and BEC1011, based on the cell death assay discussed below, also suggests that there was no cross silencing of the two BEC by either of their respective HIGS constructs, but this possibility cannot be ruled out entirely. Notably, since the expression constructs lacked encoded signal peptides, the results indicate that the proteins exert their functions from within the host cell.

To explore the possibility that one or more of the BEC is important in suppressing host defense-related cell death, we took advantage of the fact that the anthocyanin pathway in maize, barley, and wheat can be induced in leaf tissue by transiently overexpressing the $\mathrm{C} 1$ and b-Peru transcription factors (Nelson and Bushnell 1997). Anthocyanin accumulation occurs only in intact vacuoles of viable cells not affecting fungal penetration and proliferation. Co-transformation with $B$-Peru/C1-expression plasmid and RNAi vectors have been used to evaluate the potential effect of gene silencing on cell death. For example, Eichmann and associates (2010) used this system to test the effect of silencing the barley susceptibility factor (BAX inhibitor 1) on cell death. This system was applied to the eight selected BEC. Results obtained showed that the number of cells accumulating anthocyanin pigments drastically drop for BEC1011 silenced cells after $B$. graminis inoculation, revealing that BEC1011 might be involved in suppressing pathogen-induced host cell death.

In BEC1011 and BEC1054, the key active site amino acids necessary for ribonuclease activity (Nishikawa et al. 1987) are not conserved, strongly suggesting that these proteins are not, in fact, functional ribonucleases (Pedersen et al. 2012). Their roles in infection may relate to host RNA binding or to an altogether different molecular function that evolved on the ribonuclease scaffold. The inherent stability of the ribonuclease fold (Klink et al. 2000) may have been an advantage of this class of protein, particularly suited to interorganismal translocation. Though the precise molecular functions of BEC1011 and BEC1054 remain to be elucidated, they represent an intriguing new class of microbial effectors homologous to ribonuclease important for plant pathogenesis.

\section{MATERIALS AND METHODS}

\section{Plants and fungi.}

Barley seedlings (Hordeum vulgare cv. Golden Promise) were germinated at $20^{\circ} \mathrm{C}, 60 \%$ relative humidity, and $16-\mathrm{h}$ photoperiod under $190 \mu \mathrm{mol} \mathrm{m} \mathrm{m}^{-2} \mathrm{~s}^{-1}$ in a plant growth cabinet (Sanyo Gallenkamp PLC, Loughborough, U.K.). B. graminis f. sp. hordei isolates IM82 and CH4.8 were propagated on 7-dayold 'Golden Promise' seedlings by dusting B. graminis conidia derived from 7-day-old previously infected seedlings.

For HIGS experiments, 'Golden Promise' barley seedlings were grown in pots containing soil without fertilizer. Seven leaves of 7-day-old plants were used for particle bombardment experiments. The pathogen isolate B. graminis $\mathrm{CH} 4.8$ was maintained on barley seedlings in separate chambers at $20^{\circ} \mathrm{C}$ with a 16-h photoperiod under $190 \mu \mathrm{mol} \mathrm{m} \mathrm{m}^{-2} \mathrm{~s}^{-1}$ in a plant growth cabinet (Sanyo).

Seven days after seedling inoculation, B. graminis conidia were used for the inoculation of detached leaves by shaking diseased plants inside an inoculation box.

\section{Microscopy.}

B. graminis conidia and infection structures shown in Figure 2 were observed by optical microscopy using various techniques as follows. For Figure 2A, conidia were suspended in $0.1 \%$ Tween-20 in water and were imaged using conventional differential interference contrast microscopy. For Figure 2B, conidia were allowed to germinate on hydrophobic glass and were observed by bright field microscopy $12 \mathrm{~h}$ after germination. For Figure 2C through $\mathrm{F}$, segments from infected barley leaves were incubated in $1 \%$ trypan blue in ethanolic lactophenol at $95^{\circ} \mathrm{C}$ for $10 \mathrm{~min}$. The dye was then removed and the leaf segments were destained by incubating in chloral hydrate $(2 \mathrm{~g} / \mathrm{ml})$ and changing the solution until the leaf tissues where clear. For Figure $2 \mathrm{G}$, sporulating colonies of $B$. graminis were viewed under a dissecting microscope and a series of photomicrographs were taken at different focal planes and stacked using Helicon Focus software to obtain an image with increased depth of field. For Figure $2 \mathrm{H}$, epiphytic B. graminis structures were removed from infected barley leaves, and the epidermis was then dissected; fungal structures were then stained using Alexa 488-wheat germ agglutinin as previously described (Both et al. 2005).

\section{RNA extraction and cDNA synthesis.}

For RNA extraction, three to four leaves of 5-day-old infected barley seedlings were ground to fine powder with liquid nitrogen by using a precooled mortar and pestle. RNA was extracted using guanidine isothiocyanate, as previously described by Both and colleagues (2005), and was further purified using RNAClean XP beads (Beckman Coulter Ltd., High Wycombe, U.K.). Poly (A)+ RNA was treated with DNase and reverse transcribed by oligo (dT) and random priming into cDNA using the iScript synthesis kit (Bio-Rad, Hemel Hempstead, U.K.).

\section{Plasmids used in this study.}

The vector pCR8 was used as pEntry vector for the Gateway cloning system (Invitrogen/Life Technologie, Carlsbad, CA, U.S.A.). Plasmid pTA30 was developed by Douchkov and associates (2005) as a Gateway destination vector to direct highly efficient RNAi. The vector is based on a pUC18 backbone, Cauliflower mosaic virus (CaMV) 35S promoter and terminator and two Gateway cassettes containing attR sites that are oriented in an opposite direction and separated by the second intron of the wheat RGA2. It also contains the $c c d B$ gene and an ampicillin-resistance gene. Plasmid pIPKTA9 was developed by Zimmermann and associates (2006), it is a pUC18derived vector containing the CaMV $35 \mathrm{~S}$ promoter and terminator and was used for transient overexpression of wobble synthetic genes in barley leaves. This vector is not compatible with the Gateway system. Plasmid pUbiGUS, previously described by Schweizer and associates (2000), was used as reporter gene for transformed epidermal cells. It contains the GUS gene under the control of the maize ubiquitin promoter and CaMV 35S terminator. Plasmid pBC17 previously described by Leduc and colleagues (1994) carries the maize genes B-Peru and $C 1$, both under the control of a CaMV $35 \mathrm{~S}$ promoter, for anthocyanin production. This plasmid was used to assess cell viability in cell death assays.

\section{Primer design and preparation of RNAi constructs.}

Gene-specific primers were designed for each specific candidate effector gene to amplify the complete coding sequence (CDS) excluding the signal peptide sequence predicted by SignalP 4.0 server. PCR reactions were performed in a total volume of $50 \mu \mathrm{l}$ by using either a high fidelity Taq (AccuPrime Taq DNA polymerase high fidelity, Invitrogen) or standard Taq polymerase, which add a poly(A) tail to the $3^{\prime}$ end of amplified fragments required for further cloning. Dimethyl sulfoxide was also added to the mix, in order to avoid secondary structures between both template and primers. PCR parameters (primer and template annealing time and temperature, final extension time) were optimized for each candidate effector gene. The PCR products were purified by a MinElute UF kit (Qiagen, Hilden, Germany) and were eluted with $30 \mu \mathrm{l}$ of water. 
For fast cloning of Taq polymerase-amplified PCR products into the pENTRY PCR8 vector, the pCR8/GW/TOPO TA cloning kit (Invitrogen) was used, following the manufacturer's instructions. Primers GW1 (5'-gttgcaacaaattgatgagcaatgc-3') and GW2 (5'-gttgcaacaaattgatgagcaatta-3'), binding sites within the attL1 and attL2 regions, were used for sequencing plasmids extracted from positive colonies. CDS fragments in pCR8 were cloned into the pTA30 vector as inverted repeats by a single LR recombination reaction. For this, the Gateway LR Clonase II enzyme mix kit (Iinvitrogen/Life Technologies) was used, following the manufacturer's instructions, adjusting incubation of the recombination reaction to $4 \mathrm{~h}$. All pTA30-BEC were fully sequenced with primer binding sites within the intergenic regions (Intron I forward, 5'-aggcaactagataaccattttca-3' $3^{\prime}$ and Intron II reverse, $5^{\prime}$-ggatagccctcatagatagagtactaactaa- $3^{\prime}$ ) to avoid constructs with mutations in CDS, wrong insertion points, or both.

\section{RNAi rescue constructs.}

Synthetic BEC1011 wobble (354 bp) and BEC1054 wobble (354 bp) (Biomatik [SL], Wilmington, DL, U.S.A.) were designed by replacing the original $B$. graminis codons for the most different and commonly used $H$. vulgare subsp. vulgare codons encoding the same amino acid. Cloning of Wobble: 1011 and Wobble:1054 into pIPKTA9 overexpression vector

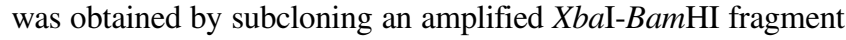
of the full CDS, excluding the signal peptide, into the $X b a \mathrm{I}$ BamHI sites of pIPKTA9.

\section{Sequence processing and analyses.}

Putative signal peptides in the deduced amino acid sequences were predicted according to the hidden Markov models using SignalP V4.0. The Phyre $^{2}$ automatic fold recognition server was used for predicting the structure, function, or both of the seven selected candidate effectors genes. Multiple alignments among sequences were performed using the slow and accurate pairwise alignment in Clustal $\mathrm{W}$, as well as the SEQMAN program from DNASTAR. Si-Fi software, a software designed for checking long, double-stranded RNAi constructs for target specificity and silencing efficiency was used for testing possible cross-silencing effects between different constructs.

\section{Quantification of candidate effector RNA during infection.}

RT-qPCR was carried out using the Platinum SYBR Green qPCR SuperMix-UDG (Invitrogen) system. The reaction included $10 \mu \mathrm{l}$ of SYBRGreen qPCR SuperMix, $9.2 \mu \mathrm{l}$ of cDNA template from each infection time point, $0.4 \mu \mathrm{l}$ of each $10 \mu \mathrm{M}$ primer, and $0.04 \mu \mathrm{l}$ of ROX reference dye added to the reaction. qPCR reactions were carried out on 96-well plates using a Applied Biosystems 7500 Fast real-time PCR system(Life Technologies, Foster City, CA, U.S.A.). The thermal cycle was split into three stages: i) $95^{\circ} \mathrm{C}$ for $20 \mathrm{~s}$, ii) 40 repetitions of $95^{\circ} \mathrm{C}$ for $30 \mathrm{~s}$, followed by $60^{\circ} \mathrm{C}$ for $30 \mathrm{~s}$, iii) (dissociation step) $95^{\circ} \mathrm{C}$ for $15 \mathrm{~s}, 60^{\circ} \mathrm{C}$ for $20 \mathrm{~s}, 95^{\circ} \mathrm{C}$ for $15 \mathrm{~s}, 60^{\circ} \mathrm{C}$ for 15 s. Results were analyzed using the $\Delta \Delta \mathrm{Ct}$ method (Livak and Schmittgen 2001). mRNA levels were normalized to that of 6$\beta$-tubulin (tub2; GenBank number, HM538443.1). The expression of each gene was calculated relative to the levels of RNA in the conidia $(0 \mathrm{hpi})$ reference that acted as the calibrator sample.

\section{Single cell assay and RNAi rescue experiments.}

HIGS cell assay was performed as previously described (Douchkov et al. 2005). Seven barley leaves were bombarded (PDS-1000/He System, Bio-Rad) to codeliver $7 \mu \mathrm{g}$ of pIKTA30-BEC together with $7 \mu \mathrm{g}$ of pUbiGUS (a plasmid expressing the GUS reporter under the control of the maize
Ubi1 promoter to monitor transformation efficiency). Barley leaves were inoculated with 150 to $200 \mathrm{~B}$. graminis spores per square millimeter, 3 days after bombardment. Two days after inoculation, leaves were stained with 5-bromo-4-chloro3-indolyl glucuronide (X-Gluc) solution and were destained with trichloroacetic acid (Douchkov et al. 2005), and the number of fully formed haustoria were measured. Finally, the HI percentage was calculated by dividing the number of transformed GUS-expressing cells with haustoria by the number of total GUS-expressing cells. Controls were performed by bombarding with the empty pTA30 silencing vector and the pUbiGUS construct.

For RNAi rescue experiments, barley leaves were co-bombarded with the same constructs as for HIGS but included either the pIPKTA9:wobble:BEC1011 or pIPKTA9:wobble:BEC1054 constructs for complementation of BEC1011 and BEC1054 HIGS, respectively. In this case controls were co-bombarded with empty pTA30, empty pIPKTA9, and pUbiGUS vectors. Five independent experiments were carried out for each different combination.

\section{Cell-death assay.}

For examination of cellular viability after HIGS, we delivered $7 \mu \mathrm{g}$ per shot of vector pTA30BEC (for BEC silencing) together with $7 \mu \mathrm{g}$ of the $\mathrm{B}$-Peru/Cl-expression plasmid $\mathrm{pBC} 17$ (Schweizer et al. 2000), for anthocyanin production, and pUbiGUS, as transformation marker, into epidermal cells of barley seedling primary leaves by particle bombardment. Half of the leaves were inoculated 1 day after transformation. Five days after transformation, the leaves were stained with X-Gluc as described above, and we counted the number of cells that had a visible GUS stain and anthocyanin accumulation. Three independent experiments were conducted with and without $B$. graminis inoculation.

\section{Statistical analysis of HIGS effects.}

A generalized linear mixed effects model was fit to the haustoria count data for both the initial HIGS screen and the BEC1011 and BEC1054 wobble experiments. For each leaf, the number of GUS cells with haustoria formation was modeled as a binomial random variable with the total number of GUS cells as the number of trials and a success probability (probability of haustoria formation) specific to each combination of experiment and BEC. The log odds of haustoria formation (the logit of the success probability) was modeled as a linear function of an intercept parameter, a BEC effect, and a random experiment effect. The random experiment effects were assumed to be independent and normally distributed with mean zero and positive variance. A quasi-likelihood approach was used to account for potential overdispersion due to natural variation from leaf to leaf. This generalized linear mixed effects model was fit using SAS PROC GLIMMIX.

The natural log of the odds of haustoria formation for a BEC divided by the odds of haustoria formation for the control was estimated for each BEC. A positive log odds ratio shows that silencing of the BEC increased susceptibility, while a negative $\log$ odds ratio indicates that silencing of the BEC increased resistance. A test for a difference between each BEC effect and the control (pTA30) (equivalently, a test for the significance of each log odds ratio) was conducted to identify BEC of interest. The Dunnett-Hsu (Dunnett 1955; Hsu 1992) correction implemented in GLIMMIX was used to adjust the $P$ values from these tests to allow for control of the probability of one or more type I errors (the familywise error rate). Thus, BEC comparisons to the control with an adjusted $P$ value no larger than alpha can be declared significant while controlling the familywise error rate at level alpha. 


\section{ACKNOWLEDGMENTS}

We thank G. Fuerst (Wise Lab) for excellent technical assistance throughout the project. This research was supported by the Biotechnology and Biological Sciences Research Council (grant BB/H001646/1), the European Union (Marie Curie Fellowship to C. Pliego), and the United States National Science Foundation (Plant Genome grant 09-22746).

\section{LITERATURE CITED}

Banerjee, B., Kurup, V. P., Phadnis, S., Greenberger, P. A., and Fink, J. N. 1996. Molecular cloning and expression of a recombinant Aspergillus fumigatus protein Asp f II with significant immunoglobulin E reactivity in allergic bronchopulmonary aspergillosis. J. Lab. Clin. Med. 127:253262.

Bindschedler, L. V., McGuffin, L. J., Burgis, T. A., Spanu, P. D., and Cramer, R. 2011. Proteogenomics and in silico structural and functional annotation of the barley powdery mildew Blumeria graminis f. sp. hordei. Methods 54:432-441.

Bindschedler, L. V., Burgis, T. A., Mills, D. J. S., Ho, J. T. C., Cramer, R., and Spanu, P. D. 2009. In planta proteomics and proteogenomics of the biotrophic barley fungal pathogen Blumeria graminis f. sp. hordei. Mol. Cell. Proteomics 8:2368-2381.

Boller, T., and Felix, G. 2009. A renaissance of elicitors: Perception of microbe-associated molecular patterns and danger signals by patternrecognition receptors. Ann. Rev. Plant Biol. 60:379-406.

Both, M., and Spanu, P. 2004. Blumeria graminis f. sp. hordei, an obligate pathogen of barley. Pages 202-218 in: Plant Pathogen Interactions, N. Talbot, ed. Blackwell Publishing, Oxford.

Both, M., Eckert, S. E., Csukai, M., Müller, E., Dimopoulos, G., and Spanu, P. D. 2005. Transcript profiles of Blumeria graminis development during infection reveal a cluster of genes that are potential virulence determinants. Mol. Plant-Microbe Interact. 18:125-133.

Cantarel, B. L., Coutinho, P. M., Rancurel, C., Bernard, T., Lombard, V., and Henrissat, B. 2009. The Carbohydrate-Active EnZymes database (CAZy): An expert resource for glycogenomics. Nucleic Acid Res. 37:D233-D238.

Dodds, P. N., and Rathjen, J. P. 2010. Plant immunity: Towards an integrated view of plant-pathogen interactions. Nat. Rev. Genet. 11:539-548.

Dong, W. B., Nowara, D., and Schweizer, P. 2006. Protein polyubiquitination plays a role in basal host resistance of barley. Plant Cell 18:33213331.

Douchkov, D., Nowara, D., Zierold, U., and Schweizer, P. 2005. A highthroughput gene-silencing system for the functional assessment of defense-related genes in barley epidermal cells. Mol. Plant-Microbe Interact. $18: 755-761$

Dunnett, C. W. 1955. A Multiple comparison procedure for comparing several treatments with a control. J. Am. Stat. Assn. 50:1096-1121.

Eichmann, R., Bischof, M., Weis, C., Shaw, J., Lacomme, C., Schweizer, P., Duchkov, D., Hensel, G., Kumlehn, J., and Hückelhoven, R. 2010. BAX INHIBITOR-1 is required for full susceptibility of barley to powdery mildew. Mol. Plant-Microbe Interact. 23:1217-1227.

Ellis, J. G., and Dodds, P. N. 2011. Showdown at the RXLR motif: Serious differences of opinion in how effector proteins from filamentous eukaryotic pathogens enter plant cells. Proc. Natl. Acad. Sci. U.S.A. 108:14381-14382.

Glawe, D. A. 2008. The powdery mildews: A review of the world's most familiar (yet poorly known) plant pathogens. Annu. Rev. Phytopathol. 46:27-51.

Godfrey, D., Bohlenius, H., Pedersen, C., Zhang, Z., Emmersen, J., and Thordal-Christensen, H. 2010. Powdery mildew and rust fungal effector candidates share N-terminal Y/F/WxC-motif. BMC Genomics 11:317.

Goldman, R..C., Sullivan, P..A., Zakula, D., and Capobianco, J..O. 1995. Kinetics of $\beta-1,3$ glucan interaction at the donor and acceptor sites of the fungal glucosyltransferase encoded by the BGL2 gene. Eur. J. Biochem. 227:372-378.

Haas, B., Kamoun, S., Zody, M., Jiang, R., Handsaker, R., and Cano, L. 2009. Genome sequence and analysis of the Irish potato famine pathogen Phytophthora infestans. Nature 461:393-398.

Hacquard, S., Joly, D. L., Lin, Y.-C., Tisserant, E., Feau, N., Delaruelle, C., Legué, V., Kohler, A., Tanguay, P., Petre, B., Frey, P., Van de Peer, Y., Rouzé, P., Martin, F., Hamelin, R. C., and Duplessis, S. 2012. A comprehensive analysis of genes encoding small secreted proteins identifies candidate effectors in Melampsora larici-populina (Poplar leaf rust). Mol. Plant-Microbe Interact. 25:279-293.

Hann, D. 2010. Bacterial virulence effectors and their activities. Curr. Opin. Plant Biol. 13:388-393.

Hoefle, C., and Huckelhoven, R. 2008. Enemy at the gates: Traffic at the plant cell pathogen interface. Cell. Microbiol. 10:2400-2407.
Hsu, J. 1992. The factor analytic approach to simultaneous inference in the general linear model. J. Comput. Graph. Stat. 1:151-168.

International Barley Genome Sequencing Consortium, Mayer, K, F., Waugh, R., Brown, J, W., Schulman, A., Langridge, P., Platzer, M., Fincher. G. B., Muehlbauer, G. J., Sato, K., Close, T. J., Wise, R. P., and Stein, N. 2012. A physical, genetic and functional sequence assembly of the barley genome. Nature 491:711-716.

Kämper, J., Kahmann, R., Bolker, M., Ma, L. J., Brefort, T., Saville, B. J., Banuett, F., Kronstad, J. W., Gold, S. E., Muller, O., Perlin, M. H., Wosten, H. A. B., de Vries, R., Ruiz-Herrera, J., Reynaga-Pena, C. G., Snetselaar, K., McCann, M., Perez-Martin, J., Feldbrugge, M., Basse, C. W., Steinberg, G., Ibeas, J. I., Holloman, W., Guzman, P., Farman, M., Stajich, J. E., Sentandreu, R., Gonzalez-Prieto, J. M., Kennell, J. C., Molina, L., Schirawski, J., Mendoza-Mendoza, A., Greilinger, D., Munch, K., Rossel, N., Scherer, M., Vranes, M., Ladendorf, O., Vincon, V., Fuchs, U., Sandrock, B., Meng, S., Ho, E. C. H., Cahill, M. J., Boyce, K. J., Klose, J., Klosterman, S. J., Deelstra, H. J., Ortiz-Castellanos, L., Li, W. X., Sanchez-Alonso, P., Schreier, P. H., Hauser-Hahn, I., Vaupel, M., Koopmann, E., Friedrich, G., Voss, H., Schluter, T., Margolis, J., Platt, D., Swimmer, C., Gnirke, A., Chen, F., Vysotskaia, V., Mannhaupt, G., Guldener, U., Munsterkotter, M., Haase, D., Oesterheld, M., Mewes, H. W., Mauceli, E. W., DeCaprio, D., Wade, C. M., Butler, J., Young, S., Jaffe, D. B., Calvo, S., Nusbaum, C., Galagan, J., and Birren, B.W. 2006. Insights from the genome of the biotrophic fungal plant pathogen Ustilago maydis. Nature 444:97-101.

Kelley, L., and Sternberg, M. 2009. Protein structure prediction on the Web: A case study using the Phyre server. Nat. Prot. 4:363-371.

Klink, T. A., Woycechowsky, K. J., Taylor, K. M., and Raines, R. T. 2000 Contribution of disulfide bonds to the conformational stability and catalytic activity of ribonuclease A. Eur. J. Biochem. 267:566-572.

Livak, K. J., and Schmittgen, T. D. 2001. Analysis of relative gene expression data using real-time quantitative PCR and the $2^{-\triangle \Lambda C T}$ method. Methods 25:402-408.

Mouyna, I., Morelle, W., Vai, M., Monod, M., Léchenne, B., Fontaine, T., Beauvais, A., Sarfati, J., Prévost, M.-C., Henry, C., and Latgé, J.-P. 2005. Deletion of GEL2 encoding for a $\beta(1-3)$ glucanosyltransferase affects morphogenesis and virulence in Aspergillus fumigatus. Mol. Microbiol. 56:1675-1688.

Nelson, A. J., and Bushnell, W. R. 1997. Transient expression of anthocyanin genes in barley epidermal cells: Potential for use in evaluation of disease response genes. Trans. Res. 6:233-244.

Nishikawa, S., Morioka, H., Kim, H. J., Fuchimura, K., Tanaka, T., Uesugi, S., Hakoshima, T., Tomita, K., Ohtsuka, E., and Ikehara, M. 1987. Two histidine residues are essential for ribonuclease T1 activity as is the case for ribonuclease A. Biochemistry 26:8620-8624.

Nowara, D., Gay, A., Lacomme, C., Shaw, J., Ridout, C., Douchkov, D., Hensel, G., Kumlehn, J., and Schweizer, P. 2010. HIGS: Host-induced gene silencing in the obligate biotrophic fungal pathogen Blumeria graminis. Plant Cell 22:3130-3141.

Nunes, C. C., and Dean, R. A. 2012. Host-induced gene silencing: A tool for understanding fungal host interaction and for developing novel disease control strategies. Mol. Plant Pathol. 13:519-529.

Pedersen, C., Ver Loren van Themaat, E., McGuffin, L. J., Abbott, J. C., Burgis, T. A., Barton, G., Bindschedler, L. V., Lu, X., Maekawa, T. Weßling, R., Cramer, R., Thordal-Christensen, H., Panstruga, R., and Spanu, P. D. 2012. Structure and evolution of barley powdery mildew effector candidates. BMC Genomics 13:694.

Petersen, T. N., Brunak, S., von Heijne, G., and Nielsen, H. 2011. SignalP 4.0: Discriminating signal peptides from transmembrane regions. Nat. Meth. 8:785-786

Quevillon, E., Silventoinen, V., Pillai, S., Harte, N., Mulder, N., Apweiler, R., and Lopez, R. 2005. InterProScan: Protein domains identifier. Nucleic Acid Res. 33:W116-W120.

Ridout, C. J., Skamnioti, P., Porritt, O., Sacristan, S., Jones, J. D. G., and Brown, J. K. M. 2006. Multiple avirulence paralogues in cereal powdery mildew fungi may contribute to parasite fitness and defeat of plant resistance. Plant Cell 18:2402-2414.

Sacristán, S., Vigouroux, M., Pedersen, C., Skamnioti, P., ThordalChristensen, H., Micali, C., Brown, J. K. M., and Ridout, C. J. 2009. Coevolution between a family of parasite virulence effectors and a class of LINE-1 retrotransposons. PLoS ONE 4:e7463. Published online.

Schirawski, J., Mannhaupt, G., Munch, K., Brefort, T., Schipper, K., Doehlemann, G., Di Stasio, M., Rossel, N., Mendoza-Mendoza, A., Pester, D., Muller, O., Winterberg, B., Meyer, E., Ghareeb, H., Wollenberg, T., Munsterkotter, M., Wong, P., Walter, M., Stukenbrock, E., Guldener, U., and Kahmann, R. 2010. Pathogenicity determinants in smut fungi revealed by genome comparison. Science 330:15461548 .

Schweizer, P., Pokorny, J., Schulze-Lefert, P., and Dudler, R. 2000. Technical advance. Double-stranded RNA interferes with gene function at 
the single-cell level in cereals. Plant J. 24:895-903.

Spanu, P. D., Abbott, J. C., Amselem, J., Burgis, T. A., Soanes, D. M., Stuber, K., van Themaat, E. V. L., Brown, J. K. M., Butcher, S. A., Gurr, S. J., Lebrun, M. H., Ridout, C. J., Schulze-Lefert, P., Talbot, N. J., Ahmadinejad, N., Ametz, C., Barton, G. R., Benjdia, M., Bidzinski, P., Bindschedler, L. V., Both, M., Brewer, M. T., Cadle-Davidson, L., CadleDavidson, M. M., Collemare, J., Cramer, R., Frenkel, O., Godfrey, D., Harriman, J., Hoede, C., King, B. C., Klages, S., Kleemann, J., Knoll, D., Koti, P. S., Kreplak, J., Lopez-Ruiz, F. J., Lu, X. L., Maekawa, T., Mahanil, S., Micali, C., Milgroom, M. G., Montana, G., Noir, S., O’Connell, R. J., Oberhaensli, S., Parlange, F., Pedersen, C., Quesneville, H., Reinhardt, R., Rott, M., Sacristan, S., Schmidt, S. M., Schon, M., Skamnioti, P., Sommer, H., Stephens, A., Takahara, H., ThordalChristensen, H., Vigouroux, M., Wessling, R., Wicker, T., and Panstruga, R. 2010. Genome expansion and gene loss in powdery mildew fungi reveal tradeoffs in extreme parasitism. Science 330:1543-1546.

Stergiopoulos, I., and de Wit, P. 2009. Fungal effector proteins. Annu. Rev. Phytopathol. 47:233-263.

Takken, F., and Rep, M. 2010. The arms race between tomato and Fusarium oxysporum. Mol. Plant Pathol. 11:309-314.
Wang, Y., and Huang, Q. 2011. Assays for effector-mediated suppression of programmed cell death in yeast. Methods Mol. Biol. 712:173-180.

Zhang, W.-J., Pedersen, C., Kwaaitaal, M., Gregersen, P. L., Mørch, S. M.

Hanisch, S., Kristensen, A., Fuglsang, A. T., Collinge, D. B., and Thordal-Christensen, H. 2012. Interaction of barley powdery mildew effector candidate CSEP0055 with the defence protein PR17c. Mol. Plant Pathol. 13:1110-1119.

Zimmermann, G., Baeumlein, H., Mock, H.-P., Himmelbach, A., and Schweizer, P. 2006. The multigene family encoding germin-like proteins of barley. Regulation and function in basal host resistance. Plant Physiol 142:181-192.

AUTHOR-RECOMMENDED INTERNET RESOURCES

Phyre $^{2}$ (Protein Homology/analogY Recognition Engine V 2.0) server: www.sbg.bio.ic.ac.uk/phyre2/html/page.cgi?id=index

SignalP 4.1 server: www.cbs.dtu.dk/services/SignalP

EMBL-EBI Clustal W server: www.ebi.ac.uk/Tools/msa/clustalw2

Labsoft's Si-Fi software: labtools.ipk-gatersleben.de 